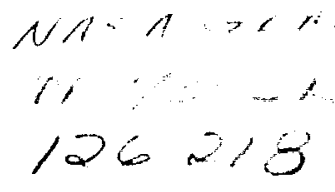

AEROSPACE REPORT NO. ATR-92(7190)-3

\title{
Ion Radial Diffusion in an Electrostatic Impulse Model for Stormtime Ring Current Formation
}

\author{
Prepared by \\ M. W. CHEN, M. SCHULZ, L. R. LYONS, and D. J. GORNEY \\ Space and Environment Technology Center \\ Technology Operations
}

15 September 1992

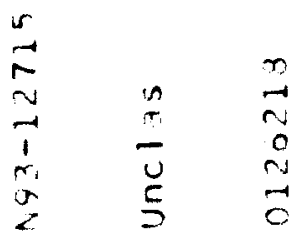

Prepared for

NASA HEADQUARTERS

Washington, DC 20546

Grant No. NAGW 2126

Engineering and Technology Group

THE AEROSPACE CORPORATION

El Segundo, California

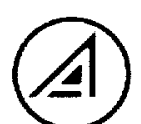

PUBLIC RELEASE IS AUTHORIZED 
Aerospace Report No.

ATR-92(7190)-3

\title{
ION RADIAL DIFFUSION IN AN ELECTROSTATIC MMPULSE MODEL FOR STORMTIME RING CURRENT FORMATION
}

\author{
Prepared by \\ M. W. Chen, M. Schulz, L. R. Lyons, and D. J. Gorney \\ Space and Environment Technology Center \\ Technology Operations
}

15 September 1992

\begin{abstract}
Engineering and Technology Group THE AEROSPACE CORPORATION

El Segundo, CA 90245-4691
\end{abstract}

Prepared for

NASA HEADQUARTERS

Washington, DC 20546

Grant No. NAGW 2126

PUBLIC RELEASE IS AUTHORIZED 
GEOPHYSICAL RESEARCH LETTERS, VOL. 19, NO. 6, PAGES 621-624, MARCH 20, 1992

\author{
ION RADIAL DIFFUSION IN AN ELECTROSTATIC IMPULSE MODEL \\ FOR STORMTIME RING CURRENT FORMATION \\ Margaret W. Chen, Michael Schulz, Larry R. Lyons, and David J. Gorney \\ Space and Environment Technology Center, The Aerospace Corporation
}

\begin{abstract}
Guiding-center simulations of stormtime transport of ring-current and radiation-belt ions having first adiabatic invariants $\mu \gtrsim 15 \mathrm{MeV} / \mathrm{G}(\mathrm{E} \gtrsim 165 \mathrm{keV}$ at $L \sim 3$ ) are surprisingly well described (typically within a factor of $\lesssim 4$ ) by the quasilinear theory of radial diffusion. This holds even for the case of an individual model storm characterized by substorm-associated impulses in the convection electric field, provided that the actual spectrum of the electric field is incorporated in the quasilinear theory. Correction of the quasilinear diffusion coeffcient $D_{L L}^{q l}$ for drift-resonance broadening (so as to define $D_{L L}^{\text {rb }}$ ) reduced the typical discrepancy with the diffusion coeftcients $D_{L L}^{\text {sim }}$ deduced from guiding-center simulations of representative-particle trajectories to a factor $\sim 3$. The typical discrepancy, was reduced to a factor $\sim 3$. The typical discrepancy was reduced to a factor
$\sim 1.4$ by averaging $D_{L L}^{s i m}, D_{L L}^{g l}$, and $D_{L L}^{r b}$ over an ensemble of model storms characterized by different (but statistically equivalent) sets of substorm-onset times.
\end{abstract}

\section{Introduction}

In a guiding-center simulation designed to investigate the access of particles to form the stormtime ring current [Chen et al., 1992], hereafter referred to as paper 1, we have found that particles having energies $E \lesssim 110 \mathrm{keV}$ could have gained access to $L \sim 3$ essentially by direct convection [Lyons and Williams, 1980] from the plasma sheet, whereas the inward stormtime transport of particles that attain energies $E \gtrsim 165 \mathrm{keV}$ at $L \sim 3$ resembles radial diffusion [cf. Cornwall, 1968; Lyons and Schulz, 1989]. We made quantitative comparisons of difusion coefficients obtained from the simulation with those derived from quasilinear diffusion theory [Falthammer, 1965] in an effort to show that the transport is indeed appropriately described as radial diffusion. The diffusion coefficients constructed from simulated particle trajectories agreed surprisingly well (typically within a factor of $\sim 4$ ) with the predictions of quasilinear radial-diffusion theory, even over a model storm whose main-phase duration (taken as $3 \mathrm{hr}$ ) amounted to only about 3-30 drift periods for representative particles of interest.

In the present work we explore two refinements which are expected to produce theoretical diffusion coefficients that agree even better with those obtained from simulated particle trajectories. The first is a resonancebroadening correction [ef. Dungey, 1965] to the quasilinear diffusion theory of $F$ älthammer [1965] for an individual model storm. The second is an application of quasilinear theory to the mean diffusion coefficients obtained by simulating particle trajectories over an ensemble of 20 statistically similar model storms.

\section{Field Model}

We use the magnetic field model that is obtained by adding a uniform southward field $\triangle B$ to the geomagnetic dipole field. This superposition leads to the appearance

Copyright 1992 by the American Geophysical Union.

Paper number 92GL00392

$0094-8534 / 92 / 92 \mathrm{GL}-00392 \$ 03.00$ of a quasi-magnetopause at the boundary between closed and open field lines. The equation of a field line in this model is

$$
\left[1+0.5(r / b)^{3}\right]^{-1}\left(r / R_{E}\right) \csc ^{2} \theta=\text { constant } \equiv L
$$

where $r$ is the geocentric distance, $\theta$ is the magnetic colatitude, $R_{E}$ is the radius of the Earth, and $b=1.5 L^{*} R_{E}=$ $12.82 R_{E}$ is the radius of the equatorial neutral line. This value of $b$ which is obtained by mapping the last closed field line (denoted $L^{*}$ ) to a colatitude of $20^{\circ}$ on the Earth, corresponds to $|\triangle B|=14.474 \mathrm{nT}$ and $L^{*}=8.547$. The limit $b \rightarrow \infty\left(L^{*} \rightarrow \infty\right)$ would correspond to a purely dipolar $B$ field. In this study, we consider only equatorially mirroring particles. The equatorial field intensity $B_{0}$ is given by $B_{0}=\left(\mu_{E} / r^{3}\right)-14.474 \mathrm{nT}$, where $\mu_{E}=3.05 \times 10^{4} \mathrm{nT}-R_{E}^{3}$ is the geomagnetic dipole moment. Further details of this field model are given by Schulz [1991, pp. 98-110].

We assume that the total electric field $\mathbf{E}=-\nabla \Phi_{E}$ is derivable from the scalar potential

$$
\Phi_{E}=-\frac{V_{\Omega}}{L}+\frac{V_{0}}{2}\left(\frac{L}{L^{*}}\right)^{2} \sin \phi+\frac{\Delta V(t)}{2}\left(\frac{L}{L^{*}}\right) \sin \phi,
$$

in which the three separate terms correspond to corotation $\left(V_{\Omega}=90 \mathrm{kV}\right)$, the Volland-Stern model of quiescent convection $\left(V_{0}=50 \mathrm{kV}\right)$, and the time-dependent enhancement $\Delta V(t)$ associated with the stormtime convection, respectively. The time-varying term in the potential is assumed to vary as $L$ rather than as $L^{2}$ because electric disturbances are expected to be less well shielded than steady-state convection by the inner magnetosphere.

We model the storm-associated enhancement $\Delta V(t)$ in the cross-tail potential drop,

$$
\Delta V(t)=\sum_{i=1}^{N} \Delta V_{i} \exp \left[\left(t_{i}-t\right) / \tau\right] \theta\left(t-t_{i}\right)
$$

where $\theta(t)$ is the unit step function ( $\equiv 1$ for $t \geq 0$; $\equiv 0$ for $t<0$ ), as a superposition of almost randomly occurring impulses that rise sharply and decay exponentially with a "lifetime" $\tau=20 \mathrm{~min}$ [cf. Cornwall, 1968]. The impulses represent the constituent substorms of a storm. The potential drop $\Delta V_{i}$ associated with any individual impulse is chosen randomly from a Gaussian distribution with a $200-\mathrm{kV}$ mean and a $50-\mathrm{kV}$ standard deviation. We assume that the $N$ start times $t_{i}$ in (3) are randomly distributed within a 3 -hr time interval corresponding to the main phase of a storm, except that we impose a 10-min "dead time" (after each impulse onset) during which no subsequent impulse can start. This constraint imposes a realistic delay between the occurrences of consecutive impulse onsets. Without such a dead-time it would be possible for the next impulse to start immediately after the previous one, and this could lead to the build-up of unrealistically large cross-tail potentials. Further details of this model storm are given in paper 1 .

We have constructed 100 such random storms so that on average there are 9 impulses per storm or 3 substorms/hr. We have randomly chosen one model storm for a detailed case study. Figure 1 shows the variation in 


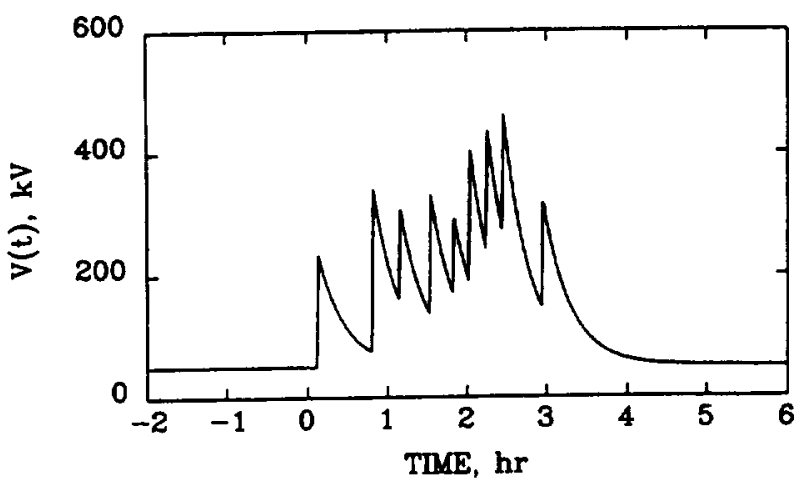

Fig. 1. Cross-tail potential $V(t)$ in our model storm.

cross-tail potential for this prototypical storm. The mean enhancement in the cross-tail potential drop for this particular storm over the time interval $t_{1}<t<t_{1}+3 \mathrm{hr}$ is $\langle\Delta V(t)\rangle=180 \mathrm{kV}$.

\section{Simulated Trajectories}

In paper 1 we considered the stormtime transport of singly charged ions with various $\mu$ values by numerically tracing their guiding-center trajectories (BurlishStoer extrapolation method). We began by tracing the quiescent trajectories obtained by setting $\Delta V(t) \equiv 0$ in (2). The solid curves in Figure 2 illustrate steady-state trajectories of equatorially mirroring ions for a particular $\mu$ value $(30 \mathrm{MeV} / \mathrm{G})$. This corresponds to an energy of $335 \mathrm{keV}$ at a geocentric radial distance of $r=3 R_{E}$. For detailed study we chose the quiescent drift shell that intersects the dusk meridian at $R \equiv r / R_{E}=3$ for each of $12 \mu$ values $(15 \mathrm{MeV} / \mathrm{G} \lesssim \mu \lesssim 200 \mathrm{MeV} / \mathrm{G})$. We selected this drift shell because it is representative of where particles need to be transported in order to form the stormtime ring current. We computed the quiescent ionic drift period $\tau_{3}$, which is $0.73 \mathrm{hr}$ for $\mu=30 \mathrm{MeV} / \mathrm{G}$. We spaced 12 representative ions equally in time on the drift path

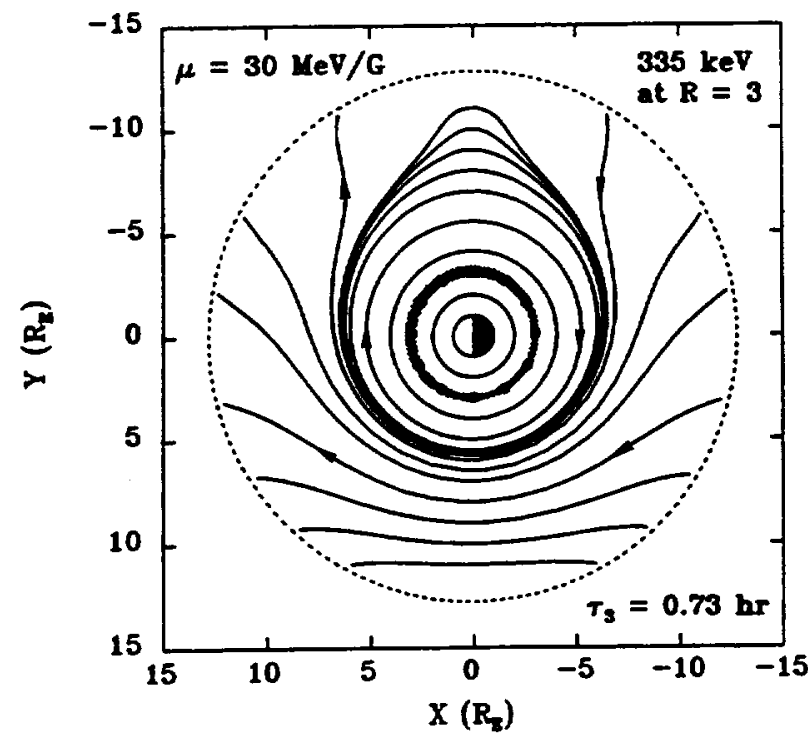

Fig. 2. Solid curves: quiet-time equatorial trajectories of singly charged ions having $\mu=30 \mathrm{MeV} / \mathrm{G}$. Outer dashed circle: neutral line at $r=b$. Small "filled" circles: the 12 representative ions' "final" positions on the steady-state drift path of interest. Stormtime trajectories computed in our time-reversed simulation span the stippled area. of interest before starting the simulation. To investigate particle access to the drift shell of interest, we ran the simulation backward in time. Thus, the time-reversed trajectories (which span the stippled area in Figure 2) indicate where the particles must have been prior to the storm in order to have reached the final drift shell $(L \approx 3)$ of interest. Indeed, we found a spread among the initial $L$ values of the representative ions. Such particle transport among closed drift paths resembles radial diffusion and was typical for ions with $\mu \gtrsim 15 \mathrm{MeV} / \mathrm{G}$ at $L \sim 3$. We also ran simulations forward in time, so as to follow the dispersal of ions from a common initial drift path. Not surprisingly, we found a qualitatively similar transport, although the particles followed in the time-forward simulations were implicitly different from those followed in the time-reversed simulations.

\section{Diffusion and Quasi-Diffusion Coefficients}

Since the quiescent drift shells are not circular (cf. Figure 2), we found it convenient to label drift shells (and thus to express the diffusion coefficients) in terms of the dimensionless third adiabatic invariant defined by Roederer $[1970$, p. 107] as

$$
\frac{1}{L} \equiv\left|\frac{\Phi_{B} R_{E}}{2 \pi \mu E}\right|=\left[\frac{1}{2 \pi} \oint \frac{d \phi}{L(\phi)}\right]
$$

where $\Phi_{B}$ is the magnetic flux enclosed by that drift shell and $L(\phi)$ denotes the field-line label at longitude $\phi$ on the drift shell. We computed diffusion coefficients for each $\mu$ from the distribution of initial $L$ values of the timereversed trajectories (see Figure 2) by constructing the quantity

$D_{L L}^{\operatorname{sim}} \equiv\left(\frac{L^{4}}{24 T}\right)\left[\sum_{i=1}^{12}\left(L_{i}^{-1}-L_{f}^{-1}\right)^{2}-\left[\sum_{i=1}^{12}\left(L_{i}^{-1}-L_{f}^{-1}\right)\right]^{2}\right]$,

where $L_{i}$ and $L_{f}$ denote the drift-shell labels of the initial and final trajectories, respectively, of the 12 representative ions and $T(=3 \mathrm{hr})$ denotes the duration of the main phase of the model storm. The quantity $D_{L L}^{s i m}$ is thus a measure of the variance among the initial third adiabatic invariants of particles situated on the final drift shell of interest. The result for each $\mu$ is plotted as an open circle in Figure 3. We also computed diffusion coefficients $D_{L L}^{\text {sim }}$ from the time-forward simulations by interchanging the indices $i$ and $f$ in (5), and these results are shown as filled circles in Figure 3. The diffusion coefficients $D_{L L}^{\text {sim }}$ obtained from simulations run forward and backward in time are not very different, $80 \%$ of them being within $\mathbf{2 4 \%}$ of each other, although they pertain implicitly to different sets of particles.

We compare the diffusion coefficients obtained from the simulated trajectories with the resonant-particle formulation [Fälthammar, 1965] of radial-diffusion theory in which the diffusion coefficient is of the form

$$
D_{L L}^{q l}=\left(L^{6} R_{E}^{4} / 4 \mu_{E}^{2}\right) \hat{E}\left(\frac{\Omega_{3}}{2 \pi}\right) \text {, }
$$

where $\hat{E}(\omega / 2 \pi)$ is the spectral-density of the (quasi-uniform) equatorial electric field in the inner magnetosphere and $\Omega_{3} / 2 \pi$ is the particles' quiescent drift frequency. When we substitute the spectral-density function for our model storm (see paper 1 for derivation) into (6), we obtain the quasilinear diffusion coefficient

$$
D_{L L}^{q l}=\frac{\tau^{2} L^{6} R_{E}^{2}}{16 T \mu_{E}^{2}\left(L^{*}\right)^{2}} \sum_{i=1}^{N} \sum_{j=1}^{N} \frac{\Delta V_{i} \Delta V_{j} \cos \left[\Omega_{3}\left(t_{j}-t_{i}\right)\right]}{1+\Omega_{3}^{2} \tau^{2}}
$$




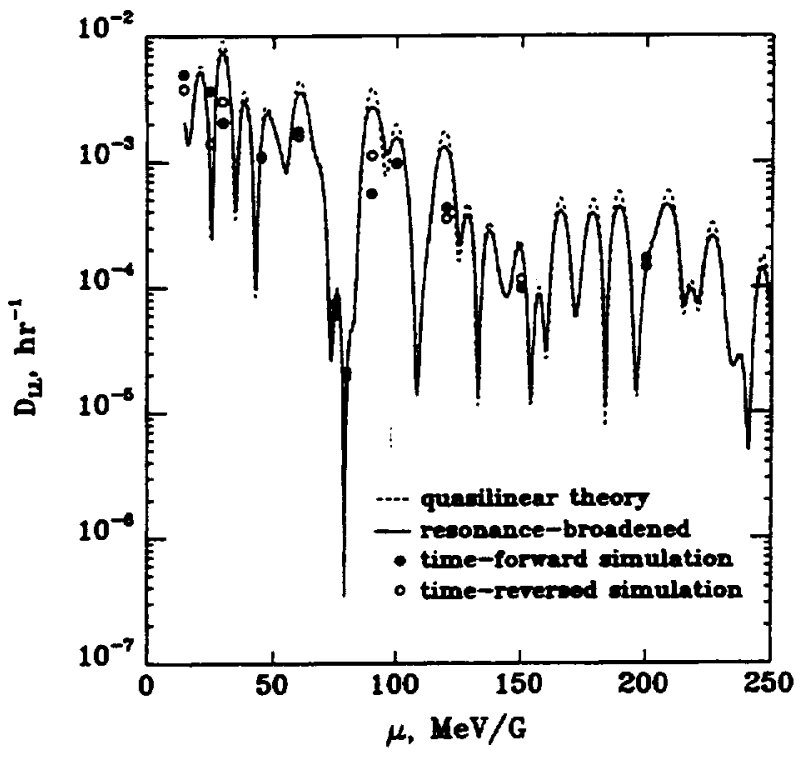

Fig. 3. Diffusion coefficients $D_{L L}^{s i m}$ obtained via (5) from time-reversed (open circles) and time-forward (filled circles) simulations, for, comparison with the quasilinear diffusion coefficient $D_{L L}^{g l}$ (dashed curve) given by (7) as an implicit function of $\mu$ and with $D_{L I}^{r b}$ (solid curve), which represents a correction of the quasitinear diffusion coefficient for resonance-broadening effects.

in which correlations between the impulses lead to cross terms $(j \neq i)$. If we neglect the cross terms, we recover essentially the standard diffusion coefficient of Cornwall [1968].

The quasilinear diffusion coefficient at $L \approx 3$, represented by the dashed curve in Figure 3, is not a very smooth function of $\mu$. This is because the impulse onsets (i) associated with any individual storm modeled by (3) occur at specific (although randomly determined) times $t_{i}$ so that the corresponding spectral density $\hat{E}(\omega / 2 \pi)$ is not a very smooth function of frequency. The diffusion coefficients obtained from the simulations can be seen to agree surprisingly well with the quasilinear results despite the strong variability of $D_{L L}^{q^{t}}$ with $\mu$. Typically (i.e., in $>80 \%$ of the available comparisons) we find agreement within a factor of 4. Quasilinear theory even accounts for the $\mu$ values (e.g., $\mu=75$ and $80 \mathrm{MeV} / \mathrm{G}$ ) for which the diffusion coefficients computed from the simulation are especially small.

However, the diffusion coefficients obtained from the simulated trajectories do not show quite as much variability with $\mu$ as quasilinear theory predicts. This is not surprising since quasilinear theory postulates a perfectly sharp resonance at the quiescent drift frequency, whereas the simulated transport leads to an eventual spread among the quiescent drift frequencies of the representative ions for each $\mu$. A rough estimate for the anticipated spread in $\Omega_{3} / 2 \pi$ is

$$
\Delta \omega / 2 \pi \approx\left(D_{L L} T / 2 \pi^{2}\right)^{1 / 2}\left|\left(\partial \Omega_{3} / \partial L\right)_{\mu}\right|
$$

since the mean-square spread in $L$ is $2 D_{L L} T$. An estimate for the diffusion coefficient (corrected for resonancebroadening effects) is thus

$$
D_{L L} \approx \frac{L^{6} R_{E}^{4}}{4 \mu_{E}^{2} \Delta \omega} \int_{\Omega_{3}-(\Delta \omega / 2)}^{\Omega_{3}+(\Delta \omega / 2)} \hat{E}\left(\frac{\omega}{2 \pi}\right) d \omega .
$$

Since the frequency bandwidth given by (8) depends on $D_{L L}$, we have iterated between (9) and (8) until satisfactory convergence to the desired solution (called $D_{L L}^{r^{b}}$ ) is achieved. The result is plotted as a solid curve in Figure 3. We find that the inclusion of nonlinear resonancebroadening effects reduces the discrepancy between quasi-r linear theory with $D_{L L}^{\text {sim }}$ through a smoothing of the sharp relative maxima and minima (with respect to $\mu$ ). Corrections were typically $\sim 10-30 \%$ at the relative maxima but were as much as $60 \%$ at the relative minima (e.g., near $\mu=184 \mathrm{MeV} / \mathrm{G}$ ). It is not clear whether the remaining discrepancy (typically a factor $\leq 3$ ) between $D_{L L}^{\text {rb }}$ and $D_{L L}^{\text {sim }}$ is intrinsic to this kind of study or attributable to the neglect of other nonlinear and/or quasilinear effects. A preliminary test suggests that the replacement of $L^{6}$ in $(9)$ by its transport-averaged value $\left\langle L^{6}\right\rangle$ would also be a relatively unimportant nonlinear correction. However, a quasi-linear correction for the variations of $L(\phi)$ and $\phi$ along a quiescent drift trajectory would introduce harmonic resonances $\left(\omega=m \Omega_{3}\right)$ in (9) and diminish the weight of the fundamental drift resonance $\left(\omega=\Omega_{3}\right)$. We hope to investigate this correction in a future study.

\section{Diffusion Averaged over an Ensemble of Storms}

It could be argued that quasilinear theory is more appropriately applied to an ensemble of model storms than to an individual storm. We tested this hypothesis by randomly choosing 20 different storms from among the 100 storms that we had constructed. We averaged the diffusion coefficients obtained from the simulations, standard quasilinear theory, and the resonance-broadened quasilinear theory over the 20 storms. The results are shown in Figure 4. The ensemble-averaged quasilinear diffusion coefficient $\bar{D}_{L L}^{q t}$ (dashed curve) and its resonance-broadened counterpart $D_{L L}^{\text {rb }}$ (solid curve) are considerably smoother than $D_{L L}^{d l}$ and $D_{L L}^{r b}$, respectively, for an individual storm. The ensemble-averaged diffusion coefficients $\bar{D}_{L L}^{\text {sim }}$ from the time-reversed and time-forward simulation (open circles and filled circles, respectively) generally agree much

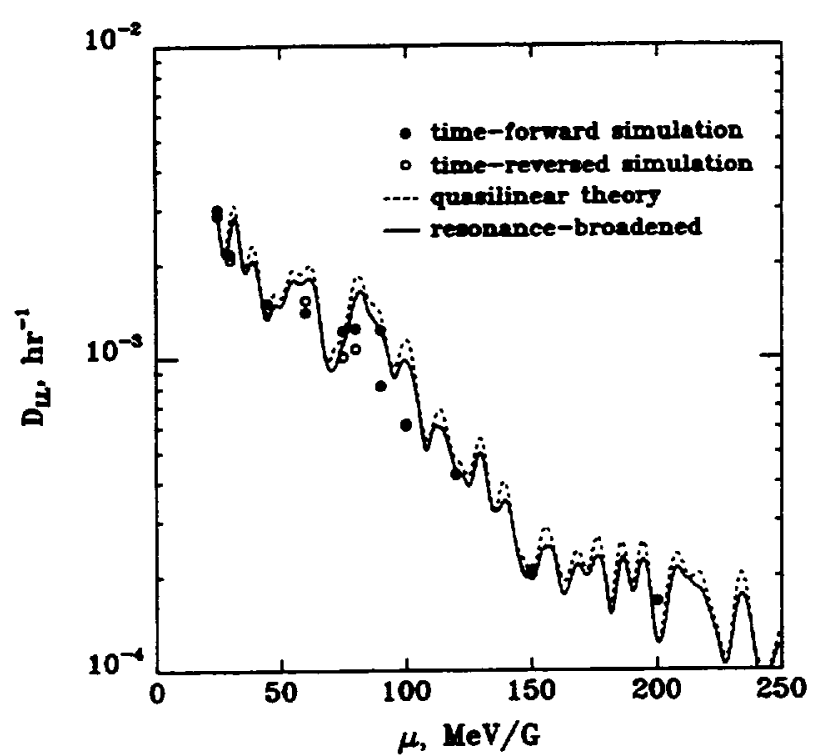

Fig. 4. Ensemble-averaged diffusion coefficients $\bar{D}_{L L}^{\text {sim }}$ obtained from the time-reversed (open circles) and timeforward (filled circles) simulations, for comparison with quasilinear theory ( $D_{L L}^{4 l}$ as a function of $\mu$, dashed curve) and with $\bar{D}_{L L}^{\text {rb }}$ (solid curve), which represents ensembleaveraged diffusion coefficient corrected for resonance broadening effects. 
better with the theoretical diffusion coefficients in Figure 4 than does $D_{L L}^{\text {sim }}$ with the theoretical diffusion coefficients for an individual storm in Figure 3. Typically $\tilde{D}_{L L}^{s i m}$ and $\tilde{D}_{L L}^{q d}$ in Figure 4 agree within a factor of only 1.4. Agreement between $\bar{D}_{L L}^{\text {sim }}$ and $\bar{D}_{L L}^{\text {rb }}$ is even better (typically within a factor of 1.3 ).

\section{Discussion and Summary}

In this work we have considered two refinements of quasilinear radial diffusion theory for the purpose of interpreting guiding-center simulation results. We found in paper 1 that the quasilinear diffusion theory of Fältham. mar [1965] already provides a surprisingly good description of charged-particle transport at first invariants $\mu \gtrsim$ $15 \mathrm{MeV} / \mathrm{G}(\mathrm{E} \gtrsim 165 \mathrm{keV}$ ) around $L=3$. This bolds even for the case of an individual model storm characterized by substorm-associated impulses in the convection electric field provided that the actual spectrum of the electric field is incorporated in the quasilinear theory.

Here we have found that a self-consistent correction of the quasilinear diffusion coefficient $D_{L L}^{q}$ for resonancebroadening effects accounts even better for the diffusion coefficients $D_{L L}$ obtained from a simulation of particle trajectories (reducing the typical discrepancy from a factor $\sim 4$ to a factor $\sim 3$ ). Resonance broadening tends to reduce the variability of $D_{L L}$ with $\mu$ by requiring that the electric spectral density $E(\omega / 2 \pi)$ be averaged over an appropriate bandwidth $\Delta \omega / 2 \pi$ surrounding the drift frequency $\Omega_{3} / 2 \pi$. The resonance-broadening correction turns out [Schulz, 1975] to be unimportant ( $\leqslant 1 \%)$ when applied to the standard model [Cormwall, 1968] of radial diffusion in which the spectral density is regarded as a smoothly varying function of frequency. However, the spectral-density for any one of our model storms is not smooth at all. Indeed, it typically shows variations of at least 2 orders of magnitude over bandwidths $\Delta \omega / 2 \pi \sim 1 \mathrm{hr}^{-1}$, whereas resonance-broadening theory typically requires $\hat{E}(\omega / 2 \pi)$ to be averaged over a bandwidth $\Delta \omega^{*} / 2 \pi \sim 0.25 \mathrm{hr}^{-1}$. The resonance-broadened correction is thus quite significant for our typical model storm.

Even better agreement between $D_{L L}^{r b}$ and $D_{L L}^{\text {sim }}$ is found when both are averaged over an ensemble of 20 model storms. Indeed, the typical discrepancy between $\bar{D}_{L L}^{\text {rb }}$ and $\bar{D}_{L L}^{\text {sim }}$ (and between $\bar{D}_{L L}^{q t}$ and $\bar{D}_{L L}^{\text {sim }}$ ) is only a factor $\sim 1.4$, mainly because $\bar{D}_{L L}^{q !}$ and $\bar{D}_{L L}^{\text {rb }} L L$ are much smoother functions of $\mu$ for an ensemble of storms than are $D_{L L}^{g t}$ and $D_{L L}^{\text {rb }}$ for an individual storm. The improved agreement achieved by ensemble-averaging is consistent with the usual interpretation of quasilinear theory (with or without the correction of resonance broadening) as a statistical description of charged-particle transport. What is surprising is that quasilinear theory provides a reasonably good description of the particle transport even for an individual storm.

Acknowledgements. Our work is supported by NASA's Space Physics Theory Program under grant NAGW-2126.
One of the authors, Margaret W. Chen, has been a National Research Council (NRC) Research Associate since the initiation of this project. We would like to thank C. T. Dum and T. H. Dupree for discussions concerning possible improvements upon our method of modeling resonance-broadening effects. Computing resources for this study were provided by the San Diego Supercomputer Center and by the NASA Center for Computational Sciences.

After having-made the ensemble-average comparison between $\bar{D}_{L L}^{s i m}$ and $\bar{D}_{L L}^{q}$, we learned of a recent similar study in which P. Riley and R. A. Wolf (preprint, 1991) have likewise found better agreement between diffusion coefficients obtained from simulated guiding-center trajectories with quasilinear diffusion theory by averaging over an ensemble of model storms than by making the comparison for an individual (in their case, actual) storm. However, they restricted their attention to particle energies $\lesssim 130 \mathrm{keV}$ at $L=3$, whereas we have found the transport to be quasi-diffusive only for particles that attain energies $\gtrsim 165 \mathrm{keV}$ at $L=3$.

\section{References}

Chen, M. W., et al., Stormtime transport of ring-current ions,J. Geophys. Res., in press, 1992.

Cornwall, J. M., Diffusion processes influenced by conjugate-point wave phenomena, Radio Sci., 9, 740-744, 1968.

Dungey, J. W., Effects of electromagnetic perturbations on particles trapped in the radiation belts, Space $S$ ci. Rev., 4, 199-222, 1965.

Fälthammar, C.-G., Effects of time-dependent electric fields on geómagnetically trapped radiation, J. Geophys. Res., 70, 2503-2516, 1965.

Lyons, L. R, and M. Schulz, Access of energetic particles to storm time ring current through enhanced radial "diffusion", J. Geophys. Res., 94, 5491-5496, 1989.

Lyons, L. R., and D. J. Williams, A source for geomagnetic storm main phase ring current, J. Geophys. Res., $85,523-530,1980$.

Roederer, J. G., Dynamics of Geomagnetically Trapped Radiation, Springer, Heidelberg, 1970.

Schulz, M., Effect of drift-resonance broadening on radial diffusion in the magnetosphere, Astrophys. Space Sci., $96,455-458,1975$.

Schulz, M., The magnetosphere, in Geomagnetism, edited by J. A. Jacobs, vol. 4, pp. 87-293, Academic Press, London, 1991.

M. W. Chen, M. Schulz, L. R. Lyons, D. J. Gorney, The Aerospace Corporation, P.O. Box 92957, Los Angeles, CA 90009 